\title{
Autocrine secretions enhance radioresistance in an exosome-independent manner in NSCLC cells
}

\author{
SHUANG WANG ${ }^{1,2^{*}}$, PIAOYANG GAO ${ }^{1 *}$, NA LI $^{1}$, PING CHEN $^{3}$, JINHAN WANG $^{1}$, \\ NINGNING HE ${ }^{1}$, KAIHUA JI ${ }^{1}$, LIQING DU ${ }^{1}$ and QIANG LIU ${ }^{1}$ \\ ${ }^{1}$ Tianjin Key Laboratory of Radiation Medicine and Molecular Nuclear Medicine, Institute of Radiation Medicine, \\ Chinese Academy of Medical Sciences and Peking Union Medical College, Tianjin 300192; \\ ${ }^{2}$ Department of Cancer Prevention Center, Tianjin Medical University Cancer Institute and Hospital, \\ National Clinical Research Center for Cancer, Key Laboratory of Cancer Prevention and Therapy, Tianjin 300060; \\ ${ }^{3}$ Department of Neurology, Inner Mongolia People's Hospital, Hohhot, Inner Mongolia 010017, P.R. China
}

Received April 25, 2018; Accepted October 1, 2018

DOI: $10.3892 /$ ijo.2018.4620

\begin{abstract}
Radiotherapy resistance in patient with non-small cell lung cancer (NSCLC) reduces patient survival and remains a significant challenge for the treatment of NSCLC. Radiation resistance has been demonstrated to be affected by secreted factors, yet it remains unclear how autocrine secretions affect the radioresistance of NSCLC cells. In the present study, the NSCLC cell line, NCI-H460, was irradiated with $\gamma$-rays (4 Gy) and then cultured in medium from $\mathrm{H} 460$ cells or normal medium to examine the potential influence of cell secretions on the radiation resistance of $\mathrm{H} 460$ cells. Cell viability, accumulation of reactive oxygen species and DNA repair capacity were all markedly improved in the irradiated
\end{abstract}

Correspondence to: Dr Liqing Du and Dr Qiang Liu, Tianjin Key Laboratory of Radiation Medicine and Molecular Nuclear Medicine, Institute of Radiation Medicine, Chinese Academy of Medical Sciences and Peking Union Medical College, 238 Baidi Road, Tianjin 300192, P.R. China

E-mail: duliqing2004@126.com

E-mail: liuqiang@irm-cams.ac.cn

"Co-first authorship

Abbreviations: CM, conditioned medium; IR, ionizing radiation; DSBs, DNA double-strand breaks; HR, homologous recombination; NHEJ, non-homologous end-joining; NSCLC, non-small cell lung cancer; ROS, reactive oxygen species; VEGF, vascular endothelial growth factor; PI3K, phosphatidylinositol-4,5-bisphosphate 3-kinase; ATM, ATM serine/threonine kinase; DMEM, Dulbecco's modified Eagle's medium; FBS, fetal bovine serum; PBS, phosphate-buffered saline; DCFH-DA, dichlorodihydrofluorescein diacetate; BSA, bovine serum albumin; SOD, superoxide dismutase; $\gamma \mathrm{H} 2 \mathrm{AX}, \gamma \mathrm{H} 2 \mathrm{~A}$ histone family member X; MRN, Mre11-Rad50-Nbs1 complex; CD9, cluster of differentiation 9; TSG101, anti-tumor susceptibility gene 101; PI, propidium iodide; p-, phosphorylated

Key words: autocrine secretions, non-small cell lung cancer, radiation resistance, reactive oxygen species, DNA repair, exosome
H460 cells that were cultured in conditioned medium (CM), compared with those cells cultured in normal medium. In addition, G2/M cell cycle arrest and upregulation of homologous recombination repair proteins were observed in the CM-treated cells, while exosomes secreted by H460 cells had no influence on the radiation resistance of H460 cells. Taken together, these results indicate that autocrine secretions enhance the radiation resistance of $\gamma$-irradiated $\mathrm{H} 460$ cells and that these secretions mainly affect the DNA repair process.

\section{Introduction}

Lung cancer is a major health problem worldwide. In China, lung cancer has become the most commonly diagnosed cancer and the leading cause of cancer-associated mortality (1). Non-small cell lung cancer (NSCLC) accounts for $85 \%$ of the lung cancer cases diagnosed worldwide (2), and for $85 \%$ of these, the cases are too advanced for surgery to be the primary treatment option on diagnosis (3). For these cases, radiotherapy is considered.

Stereotactic body radiation therapy and radiotherapy combined with chemotherapy have been demonstrated to accurately and effectively kill tumor cells, thereby prolonging patient survival (4). However, radiation resistance has been observed to develop in patients undergoing long-term radiotherapy and this restricts their treatment (5). Radiation resistance of neoplastic cells has been reported to be affected by the level of oxygen, genome stability, and the capacity of these cells to undergo DNA double-strand break (DSB) repair, proliferation and anti-apoptotic signaling (6-8). In the microenvironment of a tumor, tumor cells, fibroblasts, mesenchymal stem cells and cytokines including interleukin 6, transforming growth factor $\beta$, tumor necrosis factor are present, and all of these components can potentially contribute to the radioresistance of tumor cells $(9,10)$.

Early studies demonstrated that autocrine signaling is associated with radiation resistance $(11,12)$. In breast cancer, the autocrine cytokine, human growth hormone, may promote cell regrowth by contributing to ionizing radiation (IR)-induced resistance (13). Vascular endothelial 
growth factor (VEGF) has been documented to promote malignant astrocytoma growth and radioresistance via VEGF receptor 2 (also known as kinase insert domain receptor) and co-activation of c-Raf/mitogen-activated protein kinase, phosphatidylinositol-4,5-bisphosphate 3-kinase (PI3K)/Akt and phospholipase $\mathrm{C}$ /protein kinase $\mathrm{C}$ signaling pathways (14). In another study, the ability of cytokines to support DNA repair pathways and an ATM serine/threonine kinase-dependent DNA response were observed (15). Additionally, cell secreted exosomes, which represent extracellular microvesicles with diameters ranging from 30 to $100 \mathrm{~nm}$ that contain an abundance of proteins, messenger RNAs, microRNAs, and long non-coding RNAs, may contribute to tumor metastasis, angiogenesis and migration via autocrine/paracrine signaling (16). Recently, the role of exosomes in promoting tumor radioresistance was confirmed in head and neck cancer cells (17). Based on the results of these prior studies, it may be assumed that cells use different autocrine secretions as inducing factors to enhance their own radiation resistance and thus survival. The present study focused on the identification of changes in $\mathrm{H} 460$ cells that lead to radiation resistance in an autocrine environment.

\section{Materials and methods}

Cell cultures and $\gamma$-irradiation. The human NSCLC cell line, NCI-H460, was obtained from the Institute of Basic Medical Sciences at the Chinese Academy of Medical Sciences and Peking Union Medical College (Tianjin, China), and were cultured in Dulbecco's modified Eagle's medium (DMEM)/F12 (Hyclone; GE Healthcare Life Science, Logan, UT, USA) supplemented with $10 \%$ fetal bovine serum (FBS; Gibco; Thermo Fisher Scientific, Inc., Waltham, MA, USA). The NCI-H1299 cell line was obtained from American Type Culture Collection (Manassas, VA, USA) and was cultured in RPMI-1640 medium (Hyclone; GE Healthcare Life Science) supplemented with $10 \%$ FBS. The cells were incubated in $5 \% \mathrm{CO}_{2}$ at $37^{\circ} \mathrm{C}$. DSBs were induced in $\mathrm{H} 460$ cells by applying a ${ }^{137} \mathrm{Cs}$ Gammacell-40 radiation source (Atomic Energy of Canada Limited, Chalk River, Ontario, Canada) at a photon dose rate of $1.02 \mathrm{~Gy} / \mathrm{min}$.

Preparation of conditioned medium (CM) and exosome isolation. To prepare CM, H460 cells were grown to $80-90 \%$ confluence on $100 \mathrm{~mm}$ plates $\left(5 \times 10^{6}\right.$ cells $)$. The cells were then rinsed with phosphate-buffered saline (PBS) and incubated with DMEM/F12 containing 10\% FBS. After $48 \mathrm{~h}$, the medium was collected, membrane-filtered $(0.22 \mu \mathrm{m}$; EMD Millipore, Billerica, MA, USA) and stored at $-80^{\circ} \mathrm{C}$.

To isolate exosomes, H460 cells were incubated in serum-free DMEM/F12 until they reached $80-90 \%$ confluency. Following an additional $48 \mathrm{~h}$ of culturing, the medium was collected and then subjected to serial centrifugation at $4{ }^{\circ} \mathrm{C}(300 \times \mathrm{g}$ for $10 \mathrm{~min} ; 2,000 \mathrm{x} \mathrm{g}$ for $20 \mathrm{~min}$; and $10,000 \mathrm{x} \mathrm{g}$ for $30 \mathrm{~min})$. Following filtration of the supernatant $(0.22 \mu \mathrm{m})$, exosomes were pelleted by ultracentrifugation at $100,000 \mathrm{x}$ f for $2 \mathrm{~h}$ at $4^{\circ} \mathrm{C}$ with a $32 \mathrm{Ti}$ rotor (Optima L-100XP Ultracentrifuge; Beckman Coulter, Inc., Brea, CA, USA). The pellet was resuspended in PBS and then subjected to a second ultracentrifugation step $\left(100,000 \mathrm{x} \mathrm{g}\right.$ for $2 \mathrm{~h}$ at $\left.4^{\circ} \mathrm{C}\right)$. The pelleted exosomes were resuspended in $100 \mu \mathrm{PBS}$ and stored at $-80^{\circ} \mathrm{C}$. Bicinchoninic acid protein assays (Beyotime Institute of Biotechnology, Haimen, China) were used to determine total protein concentration for the exosome preparation.

Morphological identification of exosomes. The mixed exosomes were added to a copper net for $2 \mathrm{~min}$, and the residual liquid was removed with filter paper, which was followed by staining with $2 \%$ phosphotungstic acid solution (pH 6.4) for $2 \mathrm{~min}$ at room temperature. The prepared copper nets were then observed under a transmission electron microscope (HT77000; Hitachi, Ltd., Tokyo, Japan). Images of the exosomes were obtained at x10 magnification.

Co-culture clonogenic survival assay. To evaluate radiosensitivity, $\mathrm{H} 460$ cells were plated in 6-well plates (500 cells/well). The next day, Transwells $(0.4 \mu \mathrm{m}$; Corning Incorporated, Corning, NY, USA) containing H460 cells $\left(2.5 \times 10^{4}\right.$ cells/Transwell) were transferred into the 6 -well plates. The whole plate was then treated with ${ }^{137} \mathrm{Cs} \gamma$-radiation $(1.02 \mathrm{~Gy} / \mathrm{min})$ at variable doses $(0,2$ and $4 \mathrm{~Gy})$. In a control group, Transwells containing unirradiated $\mathrm{H} 460$ cells $\left(2.5 \times 10^{4}\right.$ cells $)$ were placed in a 6 well-plate alongside previously plated and irradiated H460 cells (500 cells/well). H1299 cells were plated in 6 -well plates $(1,000$ cells/well) and exposed to 0 , 2 or 4 Gy of $\gamma$-rays. Transwells containing unirradiated H1299 cells $\left(2.5 \times 10^{4}\right.$ cells/Transwell $)$ were subsequently added. A second set of experiments was conducted with H460 cells plated in 12 -well plates $\left(2.5 \times 10^{4}\right.$ cells/well $)$ and treated with 0 , 2 and 4 Gy irradiation. CM was collected from both irradiated and non-irradiated $\mathrm{H} 460$ cells and these samples were filtered $(0.22 \mu \mathrm{m})$ and added to the appropriate 12-well plates every $48 \mathrm{~h}$ for 7 days.

To verify the effect of exosomes on the radiosensitivity of $\mathrm{H} 460$ cells, $30 \mu \mathrm{g}$ exosomes was added to each well of a 6 -well plate containing irradiated H460 cells (18).

At 7 days after seeding, all plates were fixed with anhydrous methanol for $20 \mathrm{~min}$ and stained with $10 \%$ Giemsa stain for $30 \mathrm{~min}$, all at room temperature. The number of colonies was counted under a microscope (Nikon Eclipse TS100; Nikon Corporation, Tokyo, Japan), which a colony consisting of at least 50 cells.

Cell viability assay. H460 cells were seeded into 96-well plates $(1,500$ cells/well) and treated with $4 \mathrm{~Gy} \gamma$-irradiation. After $24 \mathrm{~h}$, the medium was replaced with varying volumes of conditioned medium $(50,100,150,200$ and $250 \mu \mathrm{l})$ and a corresponding volume of fresh medium to achieve a total volume of $300 \mu \mathrm{l}$. After $72 \mathrm{~h}$, MTT reagent (Beijing Solarbio Science \& Technology, Co., Ltd., Beijing, China) was added to each well. After $4 \mathrm{~h}$, the medium was removed and the cells were lysed with dimethyl sulfoxide to dissolve the formazan present. Absorbance values were measured at $570 \mathrm{~nm}$ with a spectrophotometric plate reader $\left(\right.$ Synergy ${ }^{\text {TM }}$ HT; BioTek Instruments, Inc., Winooski, VT, USA).

Intracellular reactive oxygen species $(R O S)$. To investigate the effect of CM on the level of ROS, H460 cells $\left(1 \times 10^{5}\right)$ were seeded into $60-\mathrm{mm}$ plates, exposed to 4 Gy of $\gamma$-rays, 
and the medium was subsequently replaced with $\mathrm{CM}$ or fresh medium. After $24 \mathrm{~h}$, the cells were stained with $5 \mu \mathrm{M}$ dichlorodihydrofluorescein diacetate (DCFH-DA) from a Reactive Oxygen Species Assay kit (Beyotime Institute of Biotechnology) for $30 \mathrm{~min}$ at room temperature and then were washed 3 times with DMEM/F12. Immunofluoresent images were obtained with a fluorescence microscope $\left(\right.$ EVOS $^{\mathrm{TM}}$; Thermo Fisher Scientific). For flow cytometry analysis, the cells were stained as above and then digested into single cell suspensions with $0.25 \%$ trypsin and washed 3 times with PBS at room temperature. Single cell suspensions were resuspended in $500 \mu \mathrm{l}$ PBS, and analyzed by flow cytometry (Mindray BriCyte E6; Mindray, Shenzhen, China).

Western blot assay. $\mathrm{H} 460$ cells were treated or untreated for 2 , 6,12 or $24 \mathrm{~h}$ with CM following exposure to 0 or $4 \mathrm{~Gy}$ irradiation. Cell extracts were subsequently collected and lysed with radioimmunoprecipitation assay lysis buffer (CWBio, Beijing, China) at $4^{\circ} \mathrm{C}$. Proteins were isolated by centrifugation at $12,000 \mathrm{x} \mathrm{g}$ for $10 \mathrm{~min}$ at $4^{\circ} \mathrm{C}$, then quantified by the bicinchoninic acid assay prior to being separated by $12 \%$ SDS-PAGE (30 $\mu \mathrm{g}$ protein per lane). Following transfer of the separated proteins to polyvinylidene difluoride membranes, they were blocked with a $1 \%$ BSA solution (Solarbio, China). All of the antibodies were diluted in a $1 \%$ bovine serum albumin (BSA) solution ( $0.1 \mathrm{~g}$ BSA in $10 \mathrm{ml}$ tris-buffered saline with Tween-20). Target proteins were probed with antibodies recognizing superoxide dismutase (SOD)-1 (1:1,000; cat. no. ab20926; Abcam, Cambridge, UK), $\gamma \mathrm{H} 2 \mathrm{~A}$ histone family member $\mathrm{X}$ $(\gamma \mathrm{H} 2 \mathrm{AX}$; 1:1,000; cat. no. 9718s; Cell Signaling Technology, Inc., Danvers, MA, USA), Rad51 recombinase (1:1,000; cat. no. sc-398587), cyclin A (1:500; cat. no. sc-271645; both from Santa Cruz Biotechnology, Inc., Dallas, TX, USA), the Mre11-Rad50-Nbs1 (MRN) complex (1:1,000; cat. no. 8344T, MRN Complex Antibody Sampler kit), phosphorylated (p)-ATM (cat. no. 5883T) and ATM (1:1,000; cat. no. 2873T; all from Cell Signaling Technology, Inc.). An analysis of the exosome protein lysates was conducted according to the same method, except that anti-cluster of differentiation 9 (CD9; 1:1,000; cat. no. ab1832-500) and anti-tumor susceptibility gene 101 (TSG101; 1:1,000; cat. no. ab125011; both from Abcam) antibodies were used. Detection of $\beta$-tubulin (1:5,000; cat. no. 66240-1-Ig) and $\beta$-actin (1:5,000; cat. no. 66009-1-Ig; both from ProteinTech Group, Inc., Chicago, IL, USA) was also performed to provide internal positive controls for the immunoblots. The primary antibodies were incubated with the membranes overnight at $4^{\circ} \mathrm{C}$. The secondary antibodies of goat anti-mouse immunoglobulin $(\mathrm{Ig}) \mathrm{G}(\mathrm{H}+\mathrm{L})$, horseradish peroxidase (HRP) conjugate (1:5,000; cat. no. SA00001-1) and goat anti-rat IgG $(\mathrm{H}+\mathrm{L})$, HRP conjugate (1:5,000; cat. no. SA00001-15; both from ProteinTech Group, Inc.) were incubated with the membranes at room temperature for $1 \mathrm{~h}$. Bound antibodies were detected with electrochemiluminescence reagents (ProteinTech Group, Inc.) and a ChemiDoc MP Imaging System (Bio-Rad Laboratories, Inc., Hercules, CA, USA). Western blot data was analyzed quantitatively with Image Lab 5.2.1 (Bio-Rad Laboratories, Inc.).

Cell cycle analysis by flow cytometry. H460 cells were treated or untreated with CM with and without irradiation (4 Gy) for
24 h. The cells were then collected and rinsed with PBS three times. The cells were fixed in $70 \%$ ethanol overnight at $-20^{\circ} \mathrm{C}$. The next day, the cells were washed three times with cold PBS and labeled with propidium iodide (PI; Beijing Solarbio Science \& Technology, Co., Ltd.) for $20 \mathrm{~min}$ at $37^{\circ} \mathrm{C}$ in the dark. The labeled cells were subsequently detected by flow cytometry (Mindray BriCyte E6) and analyzed with FlowJo v10 software (FlowJo LLC, Ashland, OR, USA).

Immunocytofluorescence. $\mathrm{H} 460$ cells $\left(4.5 \times 10^{4}\right)$ were seeded into 12-well plates containing coverslips and incubated overnight. The next day, the H460 cells were exposed to 0 or 4 Gy and cultured in normal medium or CM. After 2, 6, 12 and $24 \mathrm{~h}$, the cells were washed 3 times with PBS and fixed with $4 \%$ paraformaldehyde for $20 \mathrm{~min}$ at room temperature. After three additional rinses in PBS, the cell membranes were permeabilized with $0.3 \%$ Triton $\mathrm{X}-100$ at room temperature. After $20 \mathrm{~min}$, the cells were rinsed three times with PBS and then incubated in blocking buffer (1\% BSA) at room temperature. After $1 \mathrm{~h}$, the cells were incubated with anti- $\gamma \mathrm{H} 2 \mathrm{AX}$ antibodies (1:200) at room temperature. After an additional $2 \mathrm{~h}$, the cells were rinsed three times in PBS and then incubated with cyanine 3-conjugated anti-goat/mouse antibodies (1:200; cat. no. SA00009-1; ProteinTech Group, Inc.) at room temperature. After $1 \mathrm{~h}$, the cells were rinsed again in PBS three times and antifade mounting medium containing DAPI (Vector Laboratories, Inc., Burlingame, CA, USA) was used to mount the specimens. Imaging was performed via fluorescence microscopy.

Statistical analysis. Data are presented as the mean \pm standard deviation from at least three independent experiments, with each experiment performed in triplicate. The Student's t-test and one-way analysis of variance (ANOVA) followed by Duncan's multiple range test were applied with SPSS 13.0 (SPSS, Inc., Chicago, IL, USA) and $\mathrm{P}<0.05$ was considered to indicate a significant difference between the groups. The intracellular ROS levels were analyzed by Duncan's multiple range test and other data were analyzed by t-test.

\section{Results}

Autocrine secretions enhance the radioresistance of $H 460$ cells. To examine whether autocrine signaling improves the radioresistance of NSCLC cells, H460 colonies were co-cultured with $\mathrm{H} 460$ cells contained in Transwells (Fig. 1A). When the cells were incubated with irradiated or unirradiated H460 cells, the number of H460 colonies markedly increased following 2 or $4 \mathrm{~Gy}$ irradiation in the co-culture group, compared with in the control group (Fig. 1B and C). These data indicated that the irradiated H460 cells exhibited increased radioresistance following exposure to autocrine factors, and these factors were present independent of whether the added H460 cells had undergone irradiation. To verify whether other NSCLC cell lines also mediate this effect, H1299 cells were tested in the Transwell model. The same effect was observed (Fig. 1D).

Interactions between cells are dynamic and complex. To facilitate studies of these interactions, CM was used to simulate the effect of cell secretions on clonal formation. CM was 

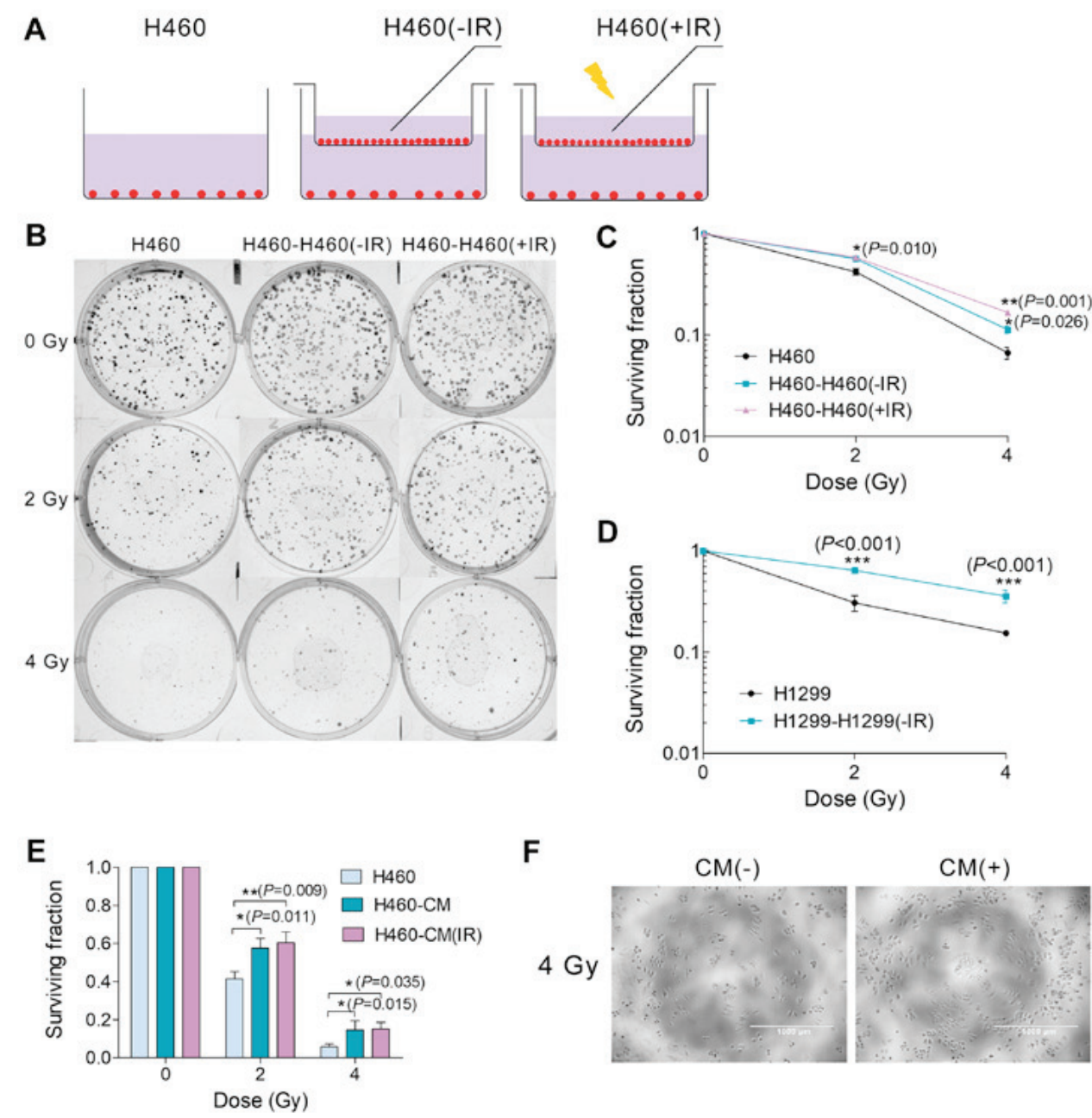

$\mathbf{F}$
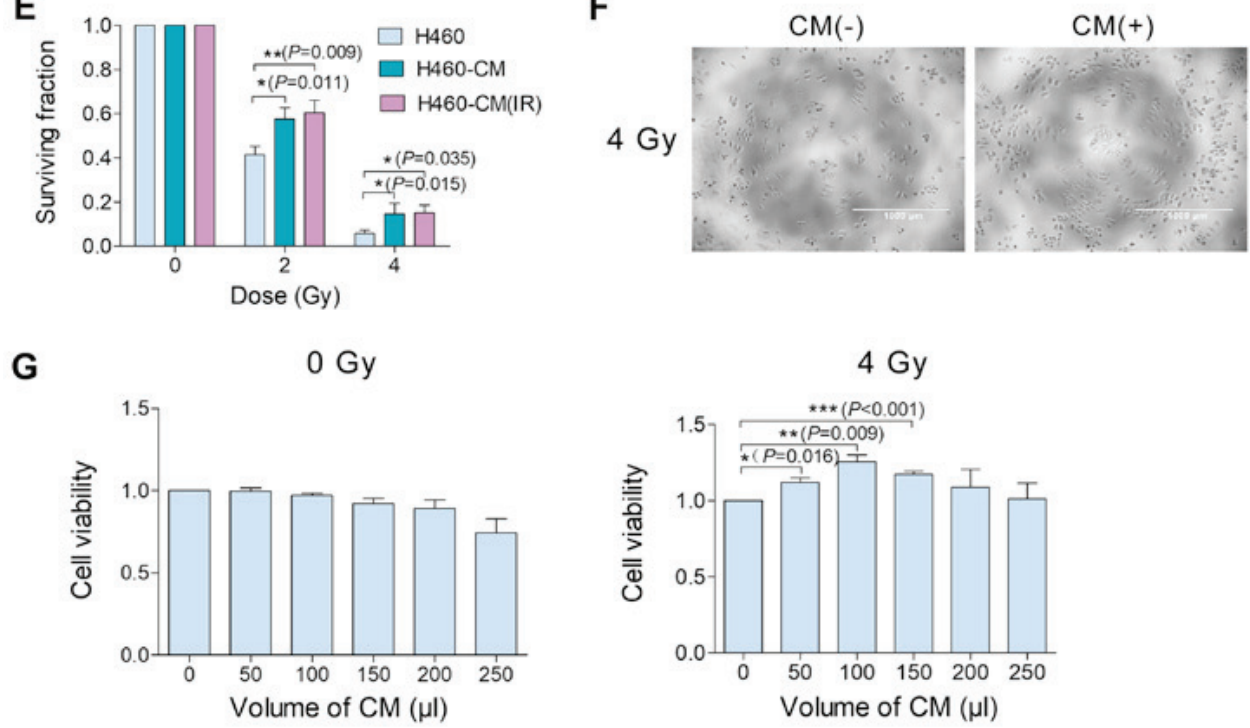

Figure 1. Autocrine secretions enhance the radioresistance of H460 cells. (A) An overview of the Transwell assay setup. Bottom: H460 cells (500 cells/well); insert: H460 cells or H460-IR cells (2.5x10 cells/well). H460-IR cells were derived from H460 cells following 2 or 4 Gy irradiation. (B and C) The effects of secretions from H460 and H460-IR cells on the colony forming capacity of H460 cells after 0, 2 or 4 Gy irradiation. (D) Survival data on the same assay in H1299 cells. (E) CM of H460 cells (with and without IR) was incubated with H460 cells after 0, 2 or 4 Gy irradiation. (F and G) Various volumes of CM from H460 cells were incubated with $\mathrm{H} 460$ cells irradiated at $4 \mathrm{~Gy}$, Magnification, $\mathrm{x} 200$. Cell viability was assessed in MTT assays 3 days later. ${ }^{*} \mathrm{P}<0.05,{ }^{* *} \mathrm{P}<0.01$, ${ }^{* * * *} \mathrm{P}<0.001$. IR, ionizing radiation; $\mathrm{CM}$, conditioned medium.

collected from $\mathrm{H} 460$ cells with or without prior irradiation, and the effects of these CM samples on the formation of $\mathrm{H} 460$ colonies following irradiation was observed (Fig. 1E). Both non-irradiated and irradiated $\mathrm{CM}$ enhanced the radiation resistance of the $\mathrm{H} 460$ cells, and these results were consistent with those obtained from the Transwell assays. Consequently, only non-irradiated $\mathrm{CM}$ was used as a relevant model in subsequent radioresistance-related studies.

Next, it was postulated that $\mathrm{H} 460$-derived CM promotes cell proliferation. When $\mathrm{H} 460$ cell viability was measured in MTT assays, the H460 cells that were irradiated and then cultured in CM exhibited greater viability compared with those cells cultured in normal medium (Fig. 1F). Interestingly, the observed effects were dependent on the volume of the CM that was applied: As the volume of CM increased, an initial increase in cell viability was observed, followed by a decrease after 4 Gy irradiation (Fig. 1G, right panel). At the higher volumes of CM, cytotoxicity appeared to be partially induced (Fig. 1G, left panel).

Autocrine secretions reduce degradation of ROS in H460 cells. To detect the level of intracellular ROS, cells were stained 
A
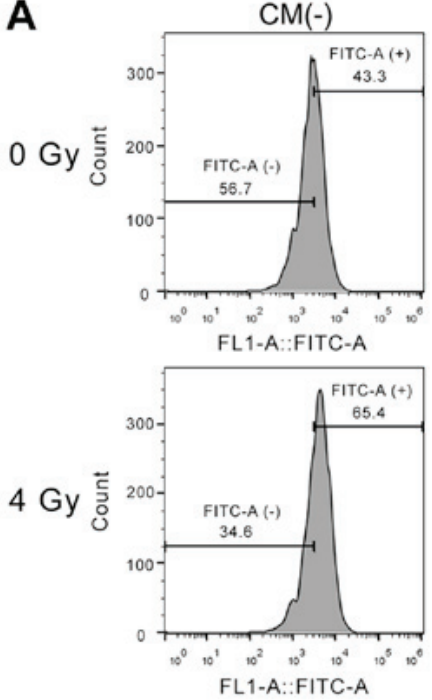

C
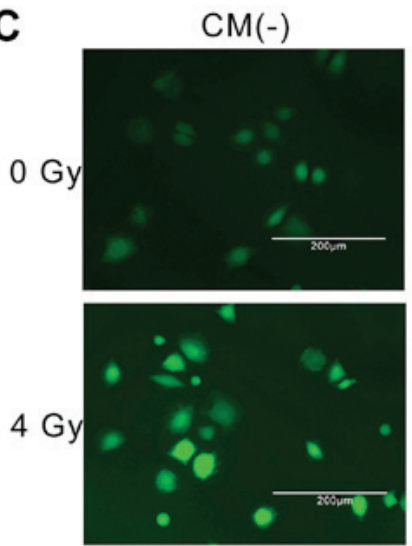
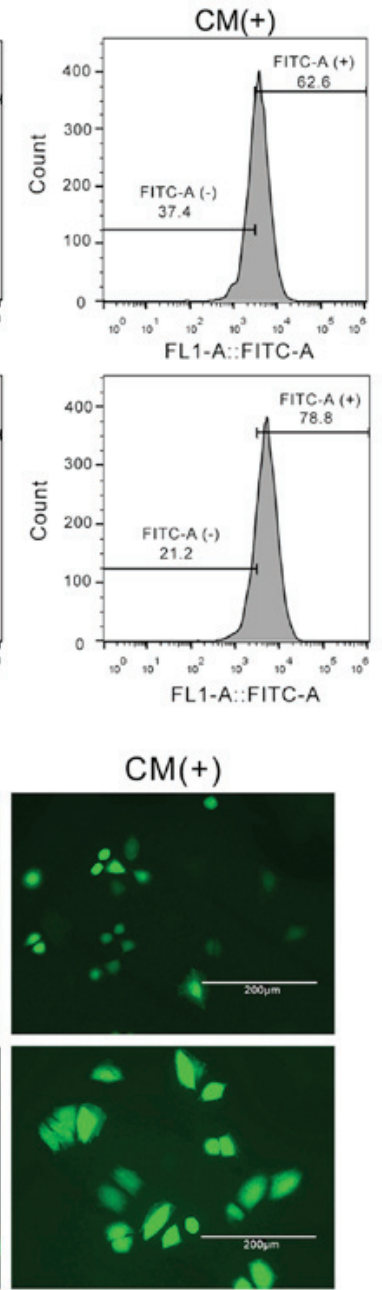

B
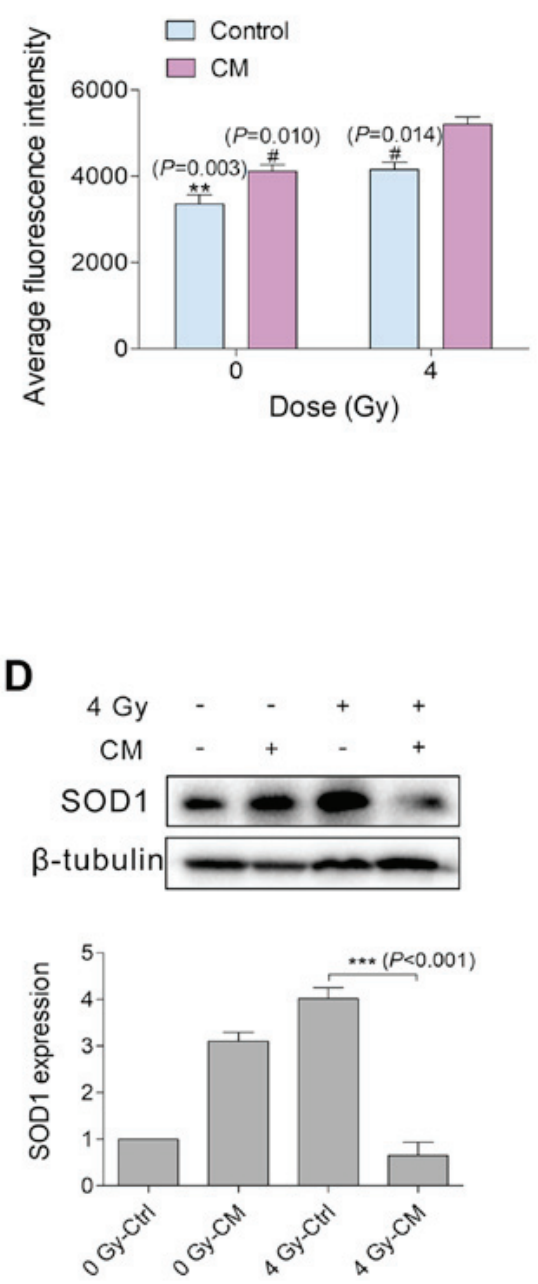

Figure 2. Autocrine secretions reduce the degradation of ROS in H460 cells. (A-D) H460 cells were incubated with or without H460 CM for 24 h after receiving 0 or $4 \mathrm{~Gy}$ irradiation. (A and C) ROS were labeled with the fluorescence indicator, dichlorodihydrofluorescein diacetate, and then detected by flow cytometry and fluorescence microscopy (magnification, x400); (B) bar graph of the average ROS levels detected in the H460 samples indicated and analyzed by Duncan's multiple range test ( $\mathrm{n}=3$ independent experiments), the $4 \mathrm{~Gy}-\mathrm{CM}$ group served as control. ${ }^{* *} \mathrm{P}<0.01,{ }^{\#} \mathrm{P}<0.05$. (D) Relative expression levels of $\mathrm{SOD} 1$ protein in the $\mathrm{H} 460$ cell samples indicated. Detection of $\beta$-tubulin served as an internal reference for normalizing target protein expression, ${ }^{* * *} \mathrm{P}<0.001$. ROS, reactive oxygen species; CM, conditioned medium; Ctrl, control; SOD1, superoxide dismutase 1.

with the fluorescence indicator, DCFH-DA. Fluorescence intensity (in arbitrary units) measured by flow cytometry demonstrated that the level of ROS increased $\sim 1.25$-fold $24 \mathrm{~h}$ after H460 cells were irradiated (4 Gy) and then cultured in CM $(5,195.87 \pm 316.76)$, compared with in those cells cultured in normal medium $(4,152.57 \pm 296.77$; Fig. 2A and B). The level of intracellular ROS was also directly observed by fluorescence microscopy. In H460 cells cultured in CM after 4 Gy irradiation, the level of intracellular ROS appeared higher than that in the normal-culture irradiated group (Fig. 2C).

The balance of intracellular ROS is regulated by various signaling molecules and signaling pathways (19). Presently, it was hypothesized that SODs may mediate the level of ROS in irradiated H460 cells. On western blot analysis, the H460 cells that were irradiated and then cultured in CM were observed to contain lower levels of SOD1, compared with in those cells cultured in normal medium (Fig. 2D). These results suggest that secretions deriving from $\mathrm{H} 460$ cells reduce the degradation of ROS in a SOD1-dependent manner.
Autocrine secretions alter the cell cycle of $H 460$ cells. To determine whether IR-treated H460 cells undergo cell cycle arrest, H460 cells were treated with 0 or 4 Gy irradiation and then cultured with CM or normal medium. After $24 \mathrm{~h}$, the cells were stained with PI. Flow cytometry detected a greater number of cells in the G2/M phase of the cell cycle among the H460 cells that were administered with 4 Gy irradiation followed by CM, compared with those H460 cells that were irradiated and then cultured in normal medium (Fig. 3A and B). To further characterize the observed cell cycle arrest, the amount of cyclin A in H460 cells with and without irradiation and subsequent culturing in $\mathrm{CM}$ was analyzed at various time points $(0,2,6,12$ and $24 \mathrm{~h})$ after irradiation. These experiments were performed based on previous reports that cyclin A is critical for the S and G2 phases of the cell cycle and is degraded in prometaphase $(20,21)$. When IR-treated H460 cells were co-cultured with the secretions of H460 cells for $2 \mathrm{~h}$, cyclin A was identified to be significantly upregulated compared with in the IR-treated, normal cultured cells at the 

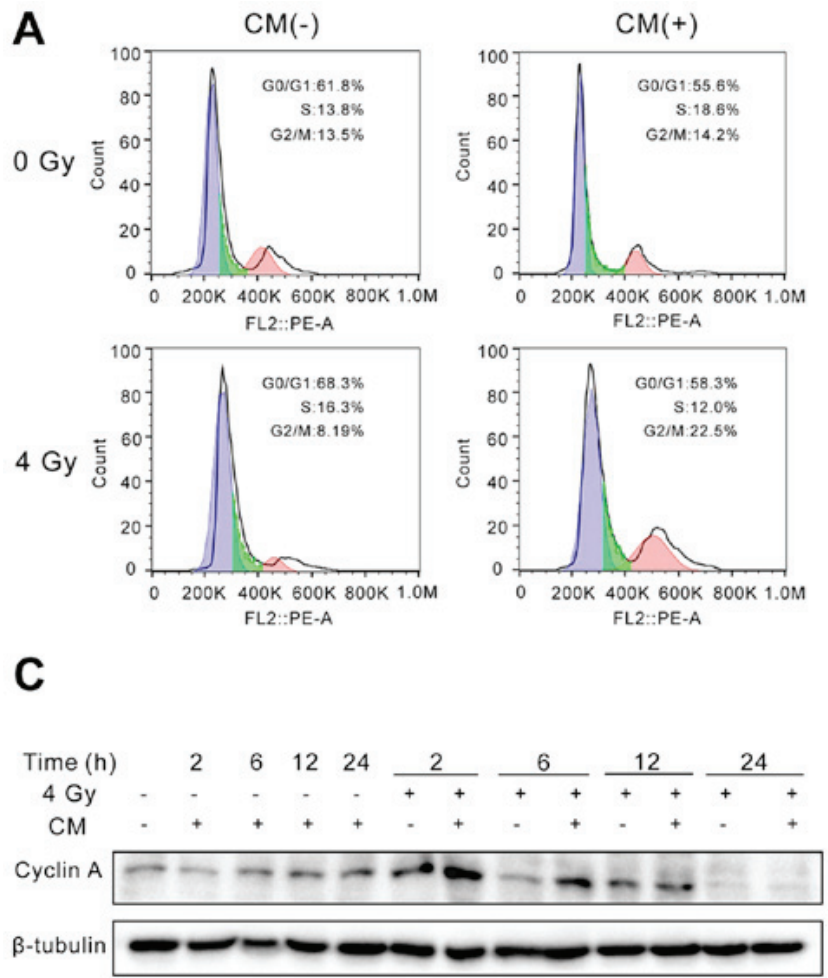

B

D

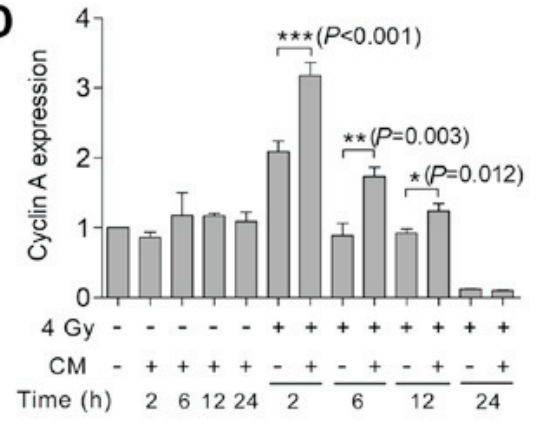

Figure 3. Autocrine secretions alter cell cycle progression in $\mathrm{H} 460$ cells. (A) $\mathrm{H} 460$ cells irradiated with 0 or 4 Gy were subject to cell cycle analysis by flow cytometry $24 \mathrm{~h}$ after treatment with H460 CM; (B) PI channel-positive cells were detected by flow cytometry and the cell cycle distribution for this population of cells was quantified. ${ }^{* *} \mathrm{P}<0.01(\mathrm{G} 2 / \mathrm{M})$. (C) Levels of cyclin A protein were detected by western blotting at 2, 6, 12 and $24 \mathrm{~h}$ after irradiation with $4 \mathrm{~Gy}$. Detection of $\beta$-tubulin served as an internal reference for normalizing target protein expression; (D) relative protein level of cyclin A in $\mathrm{H} 460$ cells was analyzed. ${ }^{*} \mathrm{P}<0.05,{ }^{* *} \mathrm{P}<0.01,{ }^{* * *} \mathrm{P}<0.001 . \mathrm{CM}$, conditioned medium.

same time point. Degradation of cyclin A was observed at the subsequent time points assayed (Fig. 3C and D).

Autocrine secretions promote DNA repair in $H 460$ cells. One of the earliest responses to DSBs is phosphorylation of histone $\mathrm{H} 2 \mathrm{AX}$ at serine $139(\gamma \mathrm{H} 2 \mathrm{AX})(22)$. This response has been reported to persist over a longer period of time in radiosensitive cell lines than in radioresistant lines (23). Here, the persistence of $\gamma \mathrm{H} 2 \mathrm{AX}$ foci was examined 2, 6, 12 and $24 \mathrm{~h}$ after $\mathrm{H} 460$ cells were treated with 4 Gy irradiation followed by culture in CM or normal medium. Among the IR-treated, CM-cultured cells, fewer positive cells were detected at the 6 $\mathrm{h}$ time point compared with in the population of IR-treated, normal cultured cells (normal medium: $80.13 \pm 1.48 \%$; CM: $62.63 \pm 2.98$; Fig. 4A and B). Detection of $\gamma \mathrm{H} 2 \mathrm{AX}$ protein by western blotting further confirmed these results (Fig. 4C). Interestingly, it was also noted that when normal $\mathrm{H} 460$ cells were treated with $\mathrm{CM}$, the content of $\gamma \mathrm{H} 2 \mathrm{AX}$ appeared to increase with time.

Homologous recombination (HR) and non-homologous end-joining (NHEJ) are the main pathways that mediate the repair of IR-induced DSBs in DNA, with the HR pathway being dominant in the $\mathrm{S}$ and G2 phases of the cell cycle (24). To examine HR repair in H460 cells, expression of Rad51 was detected in western blots. In the H460 cells that underwent irradiation and then incubation with $\mathrm{CM}$, a significant increase in Rad51 expression was observed compared with in the control cells at the 6 and $12 \mathrm{~h}$ time points following irradiation (Fig. 4D). Additionally, the Rad50, NBS1 and Mre11 proteins (which constitute the MRN complex) exhibited marked increases in expression when cultured in CM for $12 \mathrm{~h}$ and/or $24 \mathrm{~h}$ after irradiation (Fig. 4D). Meanwhile, it was observed that not only the protein content of p-ATM was increased, but also the total amount of ATM following culture in CM for 24 $\mathrm{h}$ after irradiation (Fig. 4E). These data indicate that radiation resistance in $\mathrm{H} 460$ cells is achieved with cell cycle arrest in the $\mathrm{G} 2 / \mathrm{M}$ phase and DNA repair via HR.

Exosomes from $H 460$ cells have little effect on radioresistance. The above experiments suggested that secretions from $\mathrm{H} 460$ cells reduce the radiosensitivity of IR-treated H460 cells. To determine whether exosomes contribute to this effect, exosomes were purified from H460 cell medium by differential ultra-centrifugation and then were characterized by western blotting (Fig. 5A). Exosome markers are typically identified by TSG101 and CD9 (25), but the result showed that the purified exosomes expressed the exosomal marker protein TSG101 without CD9. This may be due to the exosomes secreted by $\mathrm{H} 460$ cells not expressing CD9 protein. When the purified exosomes were visualized by transmission electron microscopy, round, bi-layer structures were observed and their diameters ranged from 30 to $100 \mathrm{~nm}$ (Fig. 5B).

Clonal formation assays were subsequently conducted with $\mathrm{H} 460$ cells that were treated with 0 or $4 \mathrm{~Gy}$ irradiation and then 
A
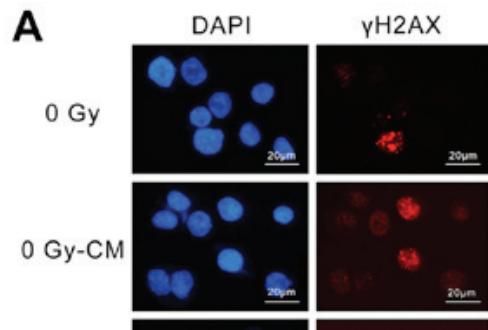

4 Gy
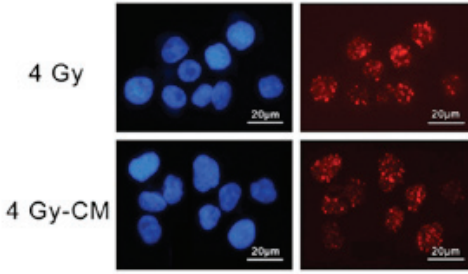

D

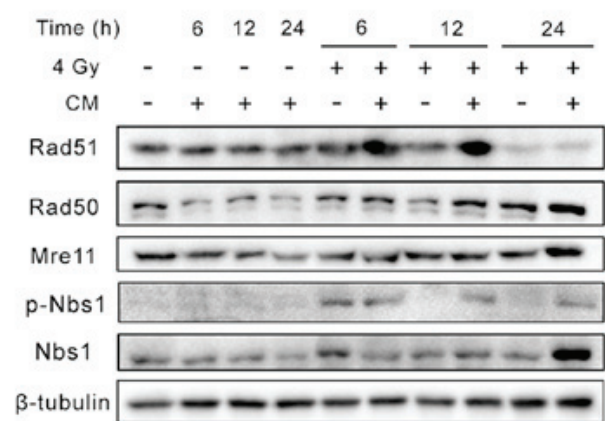

$\mathbf{F}$

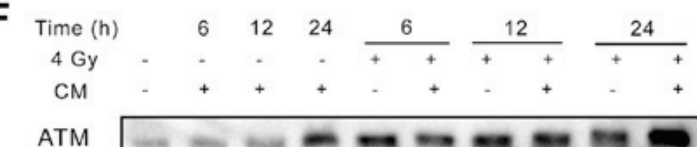

p-ATM $m m-m-m$

$\beta$-tubulin

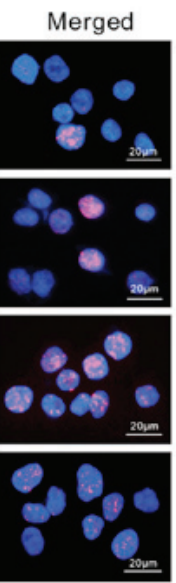

B

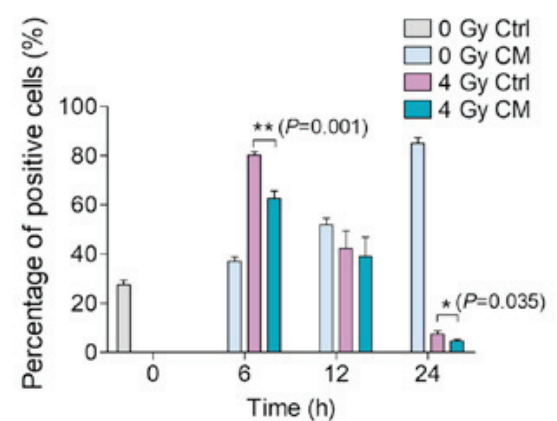

C

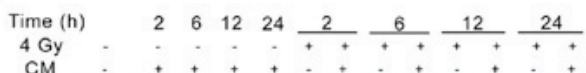

$4 \mathrm{CM}$

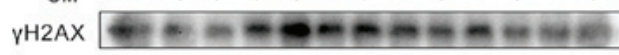

$\beta$-tubulin $--\infty-\infty-\infty$

E
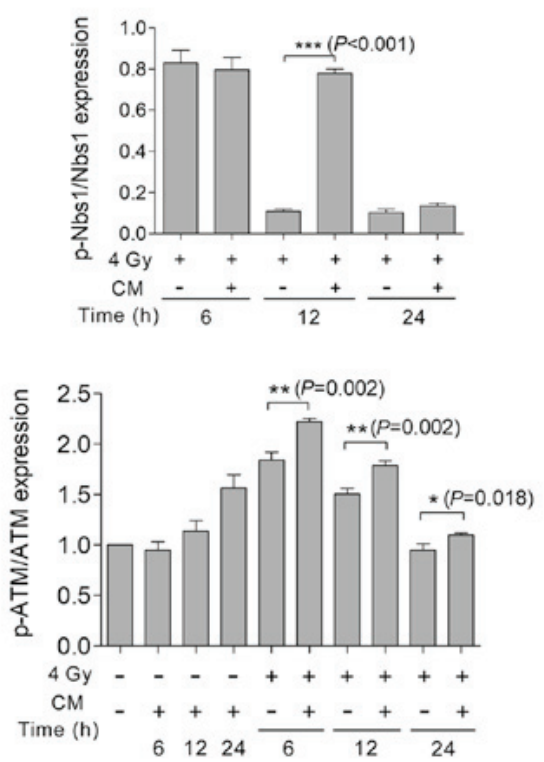

Figure 4. Autocrine secretions promote DNA repair in H460 cells. (A) Expression of $\gamma \mathrm{H} 2 \mathrm{AX}$ in $\mathrm{H} 460$ cells was detected 6 h after irradiation in immunofluorescence assays (magnification, $\mathrm{x} 400$ ). (B and C) $\mathrm{H} 460$ cells received $4 \mathrm{~Gy}$ irradiation and then were immediately incubated with $\mathrm{H} 460 \mathrm{CM}$ for 2,6 , 12 or $24 \mathrm{~h}$. The percentage of $\gamma \mathrm{H} 2 \mathrm{AX}$-positive cells $(\gamma \mathrm{H} 2 \mathrm{AX}$ foci $>10)$ was calculated and the level of $\gamma \mathrm{H} 2 \mathrm{AX}$ protein was detected by western blotting. (D) H460 cells received 4 Gy irradiation and were immediately incubated with H460 CM for 6, 12 or 24 h. Rad51, Rad50, Mre11, NBS1 and p-NBS1 in these samples were detected by western blotting. (E) Relative protein expression of p-Nbs1/Nbs1 was analyzed. (F) Levels of p-ATM and ATM in these samples were also detected by western blotting; the relative protein expression of p-ATM/ATM was analyzed. Detection of $\beta$-tubulin served as an internal reference for normalizing protein expression. ${ }^{* * *} \mathrm{P}<0.001,{ }^{* *} \mathrm{P}<0.01,{ }^{*} \mathrm{P}<0.05 . \gamma \mathrm{H} 2 \mathrm{AX}, \gamma \mathrm{H} 2 \mathrm{~A}$ histone family member $\mathrm{X}$; ATM, ATM serine/threonine kinase; p-, phosphorylated; CM, conditioned medium; Ctrl, control.

incubated with or without purified exosomes. The irradiated H460 cells that were cultured with $\mathrm{H} 460$ cell secretions in a Transwell system exhibited a significant $\sim 1.5$-fold increase in radioresistance (percent survival, $8.47 \pm 0.61 \%$ ) compared with the control cells $(5.60 \pm 1.31 \%)$. The addition of purified exosomes did not increase this effect $(8.73 \pm 0.42 \%$; Fig. $5 \mathrm{C})$. Taken together, these data suggest that exosomes secreted from $\mathrm{H} 460$ cells do not influence the radiosensitivity of H460 cells.

\section{Discussion}

In recent years, it has been demonstrated that secretions by cells in the tumor microenvironment may enhance the radiation resistance of tumor cells $(26,27)$, including recently studied exosomes (28). Regarding the relationship between
NSCLC and radiation resistance, many studies have focused on identifying internal regulatory factors of these cells $(29,30)$. In the present study, it was demonstrated that $\mathrm{H} 460$ cell-derived secretions could provide a favorable tumor microenvironment for protecting the cells against radiation effects, and that the mediating factors were not exosome-dependent.

Initially, a combination of co-culturing and typical clonal formation assays were used to verify whether secretions from $\mathrm{H} 460$ cells that enhance the radioresistance of $\mathrm{H} 460$ cells post $\gamma$-ray irradiation are independent of prior irradiation of the secreting cells. In parallel, autocrine secretions from H1299 cells were also observed to enhance the radiation resistance of H1299 cells. However, the NSCLC cell line, A549, did not exhibit the same effect (data not shown). Taken together, these results suggest that not all NSCLCs can enhance the radiation resistance of the tumor via autocrine signaling. Further 

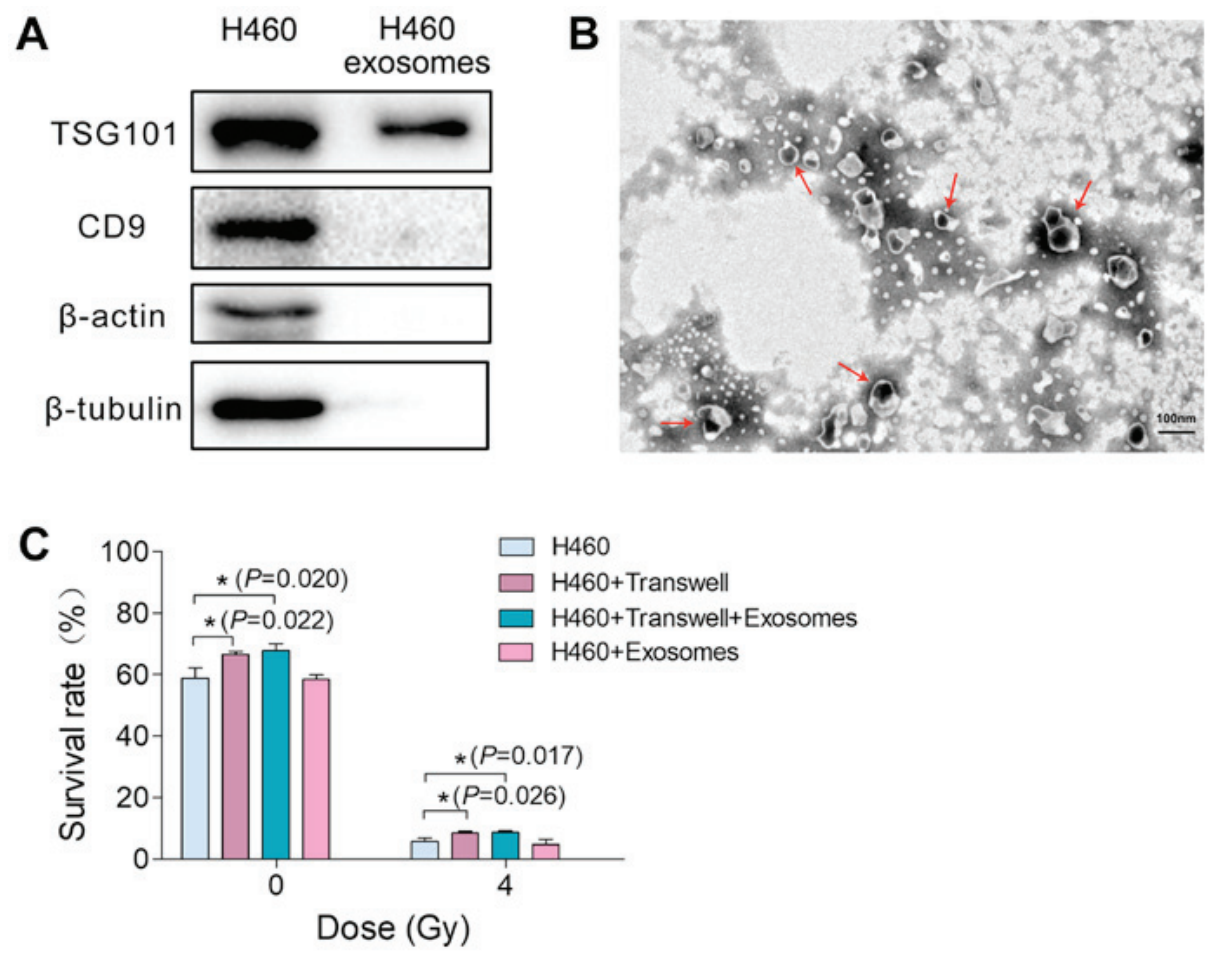

Figure 5. Exosomes isolated from $\mathrm{H} 460$ cells have little effect on $\mathrm{H} 460$ cell radioresistance. (A and B) Exosomes derived from H460 cells were characterized by (A) western blot analysis and (B) electron microscopy (red arrows indicate representative exosomes; magnification, x10,000; scale bar, 100 nm). (C) The effects of these exosomes on $\mathrm{H} 460$ cell radiosensitivity was confirmed in clonal formation assays following treatment of the $\mathrm{H} 460$ cells with 0 or $4 \mathrm{~Gy}$ irradiation ( $\mathrm{n}=3$ independent experiments). ${ }^{*} \mathrm{P}<0.05$. TSG101, anti-tumor susceptibility gene 101; CD9, cluster of differentiation 9.

experiments indicated that the observed effect was due to the promotion of cell proliferation by the secreted factors, although higher volumes of CM appeared to negatively influence cell survival. It is possible that the large quantity of cell metabolites present in the higher volumes of CM are not balanced by sufficient resources in tumor cells, and this imbalance results in cytotoxic effects. Recent studies have also demonstrated that exosomes contribute to a radiation-induced bystander effect $(31,32)$. Thus, it was hypothesized that exosomes that are produced in response to autocrine signaling could have some effect on radioresistance. However, the exosomes that were collected from H460 cells did not affect the IR-treated H460 cells in the present experiments. Therefore, it may be postulated that the autocrine substances are produced directly by $\mathrm{H} 460$ cells, and that these enhance radioresistance independently of exosomes. Moreover, this protection may not depend on the microenvironment of the cells prior to irradiation since the cells received $\mathrm{CM}$ following irradiation.

Previous study has shown that an IR-induced increase in intracellular ROS levels may cause DSBs in DNA and further induce cell death (33). The balance of kinase activity and phosphatase activity involving the PI3K/Akt pathway has been implicated in mediating the association between ROS and decreased sensitivity to induced apoptosis (34). Additionally, cancer-associated fibroblast-secreted C-X-C motif chemokine ligand 1 has been documented to inhibit the expression of the ROS-scavenging enzyme, SOD1, thereby leading to increased ROS accumulation and downstream enhanced DNA repair and radioresistance (35). In the present study, irradiated H460 cells that were cultured in $\mathrm{CM}$ were identified to have higher levels of ROS and lower levels of SOD1 than those cells in normal medium. Meanwhile, it was observed that both the ROS and SOD1 levels of non-irradiated H460 cells were increased by CM, compared with normal medium. The reason for the increase of ROS and SOD1 may be the increase of ROS production, with the increased level of SOD1 expression serving as a response to eliminate the excess ROS produced in the cells. However, when the level of ROS produced by the combination of CM and irradiation was increased, it may have exceeded the threshold of the level of ROS that can induce an increase in SOD1, manifesting as a decrease in the expression of SOD1. Based on these results, it may be hypothesized that a reduction in SOD1 degradation leads to an increase in ROS (35), thereby enhancing a cellular adaptive response and promoting cell survival. However, if the ROS content of a cell exceeds the standard adaptive response, cell death is induced.

Cell cycle arrest provides an opportunity for cells to repair their DNA. In the present study, a greater proportion of irradiated $\mathrm{H} 460$ cells incubated with $\mathrm{CM}$ were in the G2/M phase of the cell cycle. In addition, cyclin A was observed to be significantly upregulated following culture in $\mathrm{CM}$ for $2 \mathrm{~h}$ after irradiation. $\gamma \mathrm{H} 2 \mathrm{AX}$ is a marker of damage repair and may indicate the extent of DNA repair that has occurred (36). At 2-12 $\mathrm{h}$ after irradiation, the irradiated $\mathrm{H} 460$ cells cultured in $\mathrm{CM}$ were found to have significantly lower expression of $\gamma \mathrm{H} 2 \mathrm{AX}$ foci. Interestingly, longer incubation times appeared to result in increasing expression of $\gamma \mathrm{H} 2 \mathrm{AX}$ in the non-irradiated $\mathrm{H} 460$ cells. This phenomenon may be due to: i) the $\mathrm{CM}$ containing metabolites that exert cytotoxic effects on cells; ii) the CM containing secreted proteins that increase the expression of $\gamma \mathrm{H} 2 \mathrm{AX}$ in $\mathrm{H} 460$ cells; or iii) the potential for high levels of $\gamma \mathrm{H} 2 \mathrm{AX}$ expression to promote cell regeneration 
and proliferation, as previously demonstrated (37). In general, the results of the current study suggest an ability of CM from H460 cells to promote DNA repair in H460 cells treated with 4 Gy irradiation.

In radiation-resistant cells, HR serves an important role in the repair of damage due to DSBs in late $\mathrm{S} / \mathrm{G} 2$ phase (24). Rad51 is an important indicator of HR repair, and blockade of Rad51 has been demonstrated to enhance the radiosensitivity of cells (38). The findings of the present study further confirm that HR repair may promote radioresistance by enhancing the expression of Rad51 in H460 cells cultured in CM post- $\gamma$-ray irradiation. Moreover, to maintain genome stability and promote cell growth, the MRN complex participates in HR repair by activating and recruiting ATM to the DNA cleavage end, while also contributing to DNA repair and cell cycle control processes (39-41). In the present study, expression of the MRN complex and levels of p-AMT/ATM were seemingly enhanced by autocrine factors secreted by the H460 cells.

Previous study has investigated the effect of individual cytokines in the tumor microenvironment on tumor radiation resistance (15). In the present study, enhanced radioresistance of H460 cells was examined in the presence of a milieu of autocrine factors, and their effects were indicated to be independent of the presence of exosomes. Furthermore, promotion of radiation resistance appeared to be mainly mediated via enhancement of DNA repair capacity. In future studies by our group, a proteomic analysis will be conducted to further investigate the specific mechanism by which $\mathrm{H} 460$ cells exhibit enhanced radioresistance via autocrine signaling, which is anticipated to provide novel insights in the study of radioresistance mechanisms.

\section{Acknowledgements}

This study was deposited in the Dissertation Management System of Peking Union Medical College (Tianjin, China; http://dissertation.imicams.ac.cn/index.action) as part of the Master of Research thesis of Miss Shuang Wang.

\section{Funding}

The present study was supported by grants from the National Natural Science Foundation of China (grant nos. 31670859 and 81302803), the Concord Youth Fund, special funds of the Central University Basic Scientific Research Business (grant no. 3332016100), the Concord Small-Scale Characteristics of School Funding (grant no. 10023201601602) and the Chinese Academy of Medical Sciences Medical and Health Science and Technology Innovation Project (grant no. 2017-I2M-1-021).

\section{Availability of data and materials}

The analyzed data sets generated during the study are available from the corresponding author on reasonable request.

\section{Authors' contributions}

SW, LD and QL conceived and designed the study. SW, PG and NL performed the experiments. PC, JW, NH and KJ contributed to the analysis and interpretation of the data. SW wrote the paper. All authors approved the final version of the manuscript to be published.

\section{Ethics approval and consent to participate}

Not applicable.

\section{Patient consent for publication}

Not applicable.

\section{Competing interests}

The authors declare no competing interests.

\section{References}

1. Chen W, Zheng R, Baade PD, Zhang S, Zeng H, Bray F, Jemal A, Yu XQ and He J: Cancer statistics in China, 2015. CA Cancer J Clin 66: 115-132, 2016.

2. Chen Z, Fillmore CM, Hammerman PS, Kim CF and Wong KK: Non-small-cell lung cancers: A heterogeneous set of diseases. Nat Rev Cancer 14: 535-546, 2014.

3. Pathak AK, Bhutani M, Mohan A, Guleria R, Bal S and Kochupillai V: Non small cell lung cancer (NSCLC): current status and future prospects. Indian J Chest Dis Allied Sci 46: 191-203, 2004.

4. Verma V,McMillan MT, Grover S and Simone CB II: Stereotactic body radiation therapy and the influence of chemotherapy on overall survival for large ( $\geq 5$ centimeter) non-small cell lung cancer. Int J Radiat Oncol Biol Phys 97: 146-154, 2017.

5. Arechaga-Ocampo E, Lopez-Camarillo C, Villegas-Sepulveda N, Gonzalez-De la Rosa CH, Perez-Añorve IX, Roldan-Perez R, Flores-Perez A, Peña-Curiel O, Angeles-Zaragoza O, Rangel Corona $\mathrm{R}$, et al: Tumor suppressor miR-29c regulates radioresistance in lung cancer cells. Tumour Biol 39: 1010428317695010, 2017.

6. Hsieh CH, Lee CH, Liang JA, Yu CY and Shyu WC: Cycling hypoxia increases U87 glioma cell radioresistance via ROS induced higher and long-term HIF-1 signal transduction activity. Oncol Rep 24: 1629-1636, 2010.

7. Desai A, Webb B and Gerson SL: CD133+ cells contribute to radioresistance via altered regulation of DNA repair genes in human lung cancer cells. Radiother Oncol 110: 538-545, 2014.

8. Condon LT, Ashman JN, Ell SR, Stafford ND, Greenman J and Cawkwell L: Overexpression of Bcl-2 in squamous cell carcinoma of the larynx: A marker of radioresistance. Int $\mathrm{J}$ Cancer 100: 472-475, 2002.

9. Efimova E, Khodarev N, Darga T, Labay E, Levina V, Lokshin A and Weichselbaum R: Tumor radioresistance is linked to resistance to death signaling pathways associated with tumor microenvironment. In: Proceedings of the 98th AACR Annual Meeting. American Association for Cancer Research, Los Angeles, CA, 2007.

10. Chan R, Sethi P, Jyoti A, McGarry R and Upreti M: Investigating the radioresistant properties of lung cancer stem cells in the context of the tumor microenvironment. Radiat Res 185: 169-181, 2016.

11. Schlehaider U, Hill HZ, Pashapour A and Hill GJ: Influence of an autocrine multitherapy resistance factor on radiation responses of melanoma cells. Melanoma Res 4: 21-27, 1994.

12. Neta R, Perlstein R, Vogel SN, Ledney GD and Abrams J: Role of interleukin 6 (IL-6) in protection from lethal irradiation and in endocrine responses to IL-1 and tumor necrosis factor. J Exp Med 175: 689-694, 1992.

13. Bougen NM, Steiner M, Pertziger M, Banerjee A, Brunet-Dunand SE, Zhu T, Lobie PE and Perry JK: Autocrine human GH promotes radioresistance in mammary and endometrial carcinoma cells. Endocr Relat Cancer 19: 625-644, 2012.

14. Knizetova P, Ehrmann J, Hlobilkova A, Vancova I, Kalita O, Kolar Z and Bartek J: Autocrine regulation of glioblastoma cell cycle progression, viability and radioresistance through the VEGF-VEGFR2 (KDR) interplay. Cell Cycle 7: 2553-2561, 2008. 
15. Centurione L and Aiello FB: DNA Repair and Cytokines: TGF- $\beta$, IL-6, and Thrombopoietin as Different Biomarkers of Radioresistance. Front Oncol 6: 175, 2016.

16. Keller S, Sanderson MP, Stoeck A and Altevogt P: Exosomes: From biogenesis and secretion to biological function. Immunol Lett 107: 102-108, 2006.

17. Mutschelknaus L, Peters C, Winkler K, Yentrapalli R, Heider T1, Atkinson MJ and Moertl S: Exosomes derived from squamous head and neck cancer promote cell survival after ionizing radiation. PLoS One 11: e0152213, 2016.

18. Raimondo S, Saieva L, Corrado C, Fontana S, Flugy A, Rizzo A, De Leo G and Alessandro R: Chronic myeloid leukemia-derived exosomes promote tumor growth through an autocrine mechanism. Cell Commun Signal 13: 8, 2015.

19. Prasad S, Gupta SC and Tyagi AK: Reactive oxygen species (ROS) and cancer: Role of antioxidative nutraceuticals. Cancer Lett 387: 95-105, 2017.

20. Pagano M, Pepperkok R, Verde F, Ansorge W and Draetta G: Cyclin $\mathrm{A}$ is required at two points in the human cell cycle. EMBO J 11: 961-971, 1992.

21. Mateo F, Vidal-Laliena M, Canela N, Busino L, Martinez-Balbas MA, Pagano M, Agell N and Bachs $\mathrm{O}$ Degradation of cyclin A is regulated by acetylation. Oncogene 28 : 2654-2666, 2009.

22. Celeste A, Petersen S, Romanienko PJ, Fernandez-Capetillo O, Chen HT, Sedelnikova OA, Reina-San-Martin B, Coppola V, Meffre E, Difilippantonio MJ, et al: Genomic instability in mice lacking histone H2AX. Science 296: 922-927, 2002.

23. Yang JA, Liu BH, Shao LM, Guo ZT, Yang Q, Wu LQ, Ji BW, Zhu XN, Zhang SQ, Li CJ, et al: LRIG1 enhances the radiosensitivity of radioresistant human glioblastoma U251 cells via attenuation of the EGFR/Akt signaling pathway. Int J Clin Exp Pathol 8: 3580-3590, 2015.

24. Tamulevicius P, Wang M and Iliakis G: Homology-directed repair is required for the development of radioresistance during $\mathrm{S}$ phase: Interplay between double-strand break repair and checkpoint response. Radiat Res 167: 1-11, 2007.

25. Schey KL, Luther JM and Rose KL: Proteomics characterization of exosome cargo. Methods 87: 75-82, 2015.

26. Ji X, Ji J, Shan F, Zhang Y, Chen Y and Lu X: Cancer-associated fibroblasts from NSCLC promote the radioresistance in lung cancer cell lines. Int J Clin Exp Med 8: 7002-7008, 2015.

27. Chen Y, Tsai Y, Hsu JW, Duan SZ, Keng P and Lee SO: Abstract LB-210: IL-6 promotes stemness of cancer stem cells and may contribute to radiation resistance of non-small cell lung cancer. Cancer Res 74 (Suppl 19): LB-210, 2014.

28. Ma C, Nguyen H, Paradiso L, Putz U, Luwor R, Kaye A and Morokoff A: P08.35 Exosomes derived from Glioma Stem Cells (GSCs) promote cell migration, proliferation and radiation resistance in brain cancer. Neuro Oncol 19 (Suppl 3): iii61, 2017.

29. Teng K, Zhang Y,Hu X, Ding Y, Gong R and Liu L: Nimotuzumab enhances radiation sensitivity of NSCLC H292 cells in vitro by blocking epidermal growth factor receptor nuclear translocation and inhibiting radiation-induced DNA damage repair. OncoTargets Ther 8: 809-818, 2015.
30. McLaughlin KA, Nemeth Z, Bradley CA, Humphreys L, Stasik I, Fenning C, Majkut J, Higgins C, Crawford N, Holohan C, et al: FLIP: A Targetable Mediator of Resistance to Radiation in Non-Small Cell Lung Cancer. Mol Cancer Ther 15: 2432-2441, 2016.

31. Jella KK, Rani S, O'Driscoll L, McClean B, Byrne HJ and Lyng FM: Exosomes are involved in mediating radiation induced bystander signaling in human keratinocyte cells. Radiat Res 181: $138-145,2014$.

32. Xu S, Wang J, Ding N, Hu W, Zhang X, Wang B, Hua J, Wei W and Zhu Q: Exosome-mediated microRNA transfer plays a role in radiation-induced bystander effect. RNA Biol 12: 1355-1363, 2015.

33. Xie Q, Zhou Y, Lan G, Yang L, Zheng W, Liang Y and Chen T: Sensitization of cancer cells to radiation by selenadiazole derivatives by regulation of ROS-mediated DNA damage and ERK and AKT pathways. Biochem Biophys Res Commun 449: 88-93, 2014.

34. Clerkin JS, Naughton R, Quiney C and Cotter TG: Mechanisms of ROS modulated cell survival during carcinogenesis. Cancer Lett 266: 30-36, 2008.

35. Zhang H, Yue J, Jiang Z, Zhou R, Xie R, Xu Y and Wu S: CAF-secreted CXCL1 conferred radioresistance by regulating DNA damage response in a ROS-dependent manner in esophageal squamous cell carcinoma. Cell Death Dis 8: e2790, 2017.

36. Cleaver JE: $\gamma \mathrm{H} 2 \mathrm{Ax}$ : Biomarker of damage or functional participant in DNA repair 'all that glitters is not gold!'. Photochem Photobiol 87: 1230-1239, 2011.

37. Turinetto V, Orlando L, Sanchez-Ripoll Y, Kumpfmueller B, Storm MP, Porcedda P, Minieri V, Saviozzi S, Accomasso L, Cibrario Rocchietti E, et al: High basal $\gamma \mathrm{H} 2 \mathrm{AX}$ levels sustain self-renewal of mouse embryonic and induced pluripotent stem cells. Stem Cells 30: 1414-1423, 2012.

38. Chen X, Wong P, Radany EH, Stark JM, Laulier C and Wong JY: Suberoylanilide hydroxamic acid as a radiosensitizer through modulation of RAD51 protein and inhibition of homology-directed repair in multiple myeloma. Mol Cancer Res 10: 1052-1064, 2012.

39. Limbo O, Chahwan C, Yamada Y, de Bruin RA, Wittenberg C and Russell P: Ctpl is a cell-cycle-regulated protein that functions with Mre11 complex to control double-strand break repair by homologous recombination. Mol Cell 28: 134-146, 2007.

40. Helmink BA, Bredemeyer AL, Lee BS, Huang CY, Sharma GG, Walker LM, Bednarski JJ, Lee WL, Pandita TK, Bassing $\mathrm{CH}$, et al: MRN complex function in the repair of chromosomal Rag-mediated DNA double-strand breaks. J Exp Med 206: 669-679, 2009.

41. Lavin MF, Kozlov S, Gatei M and Kijas AW: ATM-Dependent Phosphorylation of All Three Members of the MRN Complex: From Sensor to Adaptor. Biomolecules 5: 2877-2902, 2015. 